\title{
\begin{tabular}{l|l} 
MitTraries & DSpace@MIT
\end{tabular}
}

\author{
MIT Open Access Articles
}

Comparison of tungsten nano-tendrils grown in Alcator C-Mod and linear plasma devices

The MIT Faculty has made this article openly available. Please share how this access benefits you. Your story matters.

Citation: Wright, G.M., D. Brunner, M.J. Baldwin, K. Bystrov, R.P. Doerner, B. Labombard, B. Lipschultz, et al. "Comparison of Tungsten Nano-Tendrils Grown in Alcator C-Mod and Linear Plasma Devices." Journal of Nuclear Materials 438 (July 2013): S84-S89.

As Published: http://dx.doi.org/10.1016/j.jnucmat.2013.01.013

Publisher: Elsevier

Persistent URL: http://hdl.handle.net/1721.1/99468

Version: Author's final manuscript: final author's manuscript post peer review, without publisher's formatting or copy editing

Terms of use: Creative Commons Attribution-Noncommercial-NoDerivatives 
PSFC/JA-12-53

\section{Comparison of tungsten nano-tendrils grown in Alcator C-Mod and linear plasma devices}

Wright, G.M., Brunner, D., Baldwin, M.J.*, Bystrov, K.**, Doerner R.P.*, Labombard, B., Lipschultz, B., de Temmerman, G.**, Terry, J.L., Whyte, D.G., Woller K.B.

* Center for Energy Research, University of California-San Diego, La Jolla, CA ** FOM Institute DIFFER, EURATOM-FOM, Trilateral Euregio Cluster, Nieuwegein, the Netherlands

January, 2013

\section{Plasma Science and Fusion Center Massachusetts Institute of Technology Cambridge MA 02139 USA}

This work was supported by the U.S. Department of Energy, Grant No. DE-SC00-02060 and DE-FC02-99ER54512. Reproduction, translation, publication, use and disposal, in whole or in part, by or for the United States government is permitted. 


\title{
Comparison of tungsten nano-tendrils grown in Alcator C-Mod and linear plasma devices
}

\author{
G.M. Wright ${ }^{\mathrm{a}}$, D. Brunner ${ }^{\mathrm{a}}$, M.J. Baldwin ${ }^{\mathrm{b}}$, K. Bystrov ${ }^{\mathrm{c}}$, R.P. Doerner ${ }^{\mathrm{b}}$, B. Labombard ${ }^{\mathrm{a}}$, B. \\ Lipschultz $^{\mathrm{a}}$, G. De Temmerman ${ }^{\mathrm{c}}$, J.L. Terry ${ }^{\mathrm{a}}$, D.G. Whyte ${ }^{\mathrm{a}}$ and K.B. Woller ${ }^{\mathrm{a}}$ \\ ${ }^{a}$ MIT Plasma Science and Fusion Center, 77 Massachusetts Ave., Cambridge, MA, USA, 02139 \\ ${ }^{b}$ Center for Energy Research, University of California in San Diego, 9500 Gilman Dr, \\ La Jolla, CA, 92093-0417, USA \\ ${ }^{c}$ FOM Institute DIFFER, Dutch Institute For Fundamental Energy Research, Association \\ EURATOM-FOM, Trilateral Euregion Cluster, Postbus 1207, 3430BE, Nieuwegein, The Netherlands
}

\begin{abstract}
:
Growth of tungsten nano-tendrils ("fuzz") has been observed for the first time in the divertor region of a high-power density tokamak experiment. After 14 consecutive helium L-mode discharges in Alcator C-Mod, the tip of a tungsten Langmuir probe at the outer strike point was fully covered with a layer of nano-tendrils. The depth of the $\mathrm{W}$ fuzz layer $(600 \pm 150 \mathrm{~nm})$ is consistent with an empirical growth formula from the PISCES experiment. Re-creating the C-Mod exposures as closely as possible in Pilot-PSI experiment can produce nearly-identical nano-tendril morphology and layer thickness at surface temperatures that agree with uncertainties with the C-Mod W probe temperature data. Helium concentrations in $\mathrm{W}$ fuzz layers are measured at 1-4 at.\%, which is lower than expected for the observed sub-surface voids to be filled with several GPa of helium pressure. This possibly indicates that the void formation is not pressure driven.
\end{abstract}

PACS: 52.40.Hf, 81.07-b, 81.05.Je, 52.55.Fa

PSI-20 keywords: Alcator C-Mod, Helium, Bubbles \& Blisters, Divertor, W fuzz

*Corresponding author address: MIT Plasma Science and Fusion Center, 77 Massachusetts Ave. NW17-121, Cambridge, MA, USA, 02139

*Corresponding author e-mail: wright@psfc.mit.edu

Presenting author: G.M. Wright 


\section{Introduction}

The growth of tungsten (W) nano-tendrils (or "fuzz") has been well documented in a wide range of linear plasma devices [1-3]. The growth conditions used for W fuzz in these devices are elevated surface temperatures $\left(\mathrm{T}_{\mathrm{s}}=1000-2000 \mathrm{~K}\right)$ of a bare tungsten substrate while implanting with helium ions at sufficient incident energies $\left(\mathrm{E}_{\mathrm{He}^{+}}>20 \mathrm{eV}\right)$. In future devices, there will be sections of the divertor that reach surface temperatures $>1000 \mathrm{~K}$ and $\mathrm{He}$ ions will always be present in D-T burning devices, thus the growth of $\mathrm{W}$ fuzz in future devices is a possibility. The impact of $\mathrm{W}$ fuzz on plasmasurface interactions and tokamak operations is still largely unknown but is expected to be significant given that fuzz layers have been grown to large depths $(>5 \mu \mathrm{m})[1]$ and therefore dramatically alter the plasma-surface interaction region. A major concern is that the growth of $\mathrm{W}$ fuzz will result in a large increase in the production of $\mathrm{W}$ dust via the mechanical failure of the individual nano-tendrils and, in turn, perhaps compromising the viability of a W divertor. Recent experiments on Alcator C-Mod have shown for the first time that $\mathrm{W}$ fuzz can be grown in a tokamak environment [4], which strengthens the argument for the existence of these structures in ITER and future fusion reactors.

Given that it has been shown to be possible to grow W fuzz in a tokamak [4], the next step is to try to gain some understanding and perspective about how these structures are forming in the tokamak. The aim of this paper is to compare the results of the W fuzz growth experiment in Alcator C-Mod to W fuzz grown in linear plasma devices with more well-known and controlled conditions and over a much broader parameter space. This comparison yields insights into the similarities and differences in nano-tendril characteristics and growth rates from fuzzy surfaces grown in a tokamak environment versus linear plasma device environment. This will help guide future work and improve extrapolations from the data set obtained on linear plasma devices towards nano-tendril growth and development in ITER and future fusion devices.

\section{Experimental}

\subsection{Alcator C-Mod exposures}


This experiment was performed in the compact, high field Alcator C-Mod tokamak $(\mathrm{R}=0.67 \mathrm{~m}$, $\mathrm{a}=0.22 \mathrm{~m}, \mathrm{~B}<8 \mathrm{~T}$ )[5]. Alcator C-Mod has high-Z refractory metals (molybdenum and tungsten) as plasma-facing surfaces in the divertor and first-wall. The goal of the experiment is to achieve the required nano-tendril growth conditions $\left(\mathrm{T}_{\mathrm{s}}=1000-2000 \mathrm{~K}, \mathrm{E}_{\mathrm{He}}>20 \mathrm{eV}\right)$ in the Alcator C-Mod lower divertor. The specific area of interest is a section of the Alcator C-Mod divertor that is intentionally ramped $\sim 2^{\circ}$ into the toroidal magnetic field lines allowing them to intercept more of the parallel heat flux, thus resulting in higher surface temperatures and stronger signals for heat flux experiments. In this section of the divertor there is a tungsten Langmuir probe that is ramped $\sim 11^{\circ}$ into the toroidal field. These Langmuir probes were in C-Mod for months of plasma operation befare the fuzz experiment, incurring 100's of seconds of plasma exposure, thus the surfaces were likely recrystallized at the time of this experiment. The strike-point was run right on top of this W Langmuir probe and it was on this probe tip that the nano-tendrils were successfully grown. The W Langmuir probe is $3 \mathrm{~mm}$ in diameter, $60 \mathrm{~mm}$ in total length, and is made from 99.95 at\% polycrystalline $\mathrm{W}$ purchased from Ed Fagan Inc. The W Langmuir probe was actively scanning from $-150 \mathrm{~V}$ to $+50 \mathrm{~V}$ in a triangle wave at $100 \mathrm{~Hz}$ during the plasma exposures. The operating conditions were an L-mode discharge with 0.9 MA of plasma current and 3.25 MW of ICRF power and a line-averaged electron density of $1.4 \times 10^{20} \mathrm{~m}^{-3}$. At the $\mathrm{W}$ Langmuir probe, $\mathrm{T}_{\mathrm{e}}$ was measured to be $\sim 25 \mathrm{eV}$. This resulted in a surface heat flux of $30-35 \mathrm{MW} / \mathrm{m}^{2}$ on the W Langmuir probe and peak surface temperatures of $\sim 2200$ K (see Fig. 1), as measured with both IR camera measurements and sheath heat flux calculations using the Langmuir probe measurements for local plasma conditions. Incident $\mathrm{He}^{+}$ion energies ranged from 50 to $250 \mathrm{eV}$ depending on the probe bias. The experiment consisted of 14 repeated discharges at these conditions which accrued $\sim 15 \mathrm{~s}$ of plasma exposure time at the appropriate conditions for fuzz growth. More details on the experimental procedure can be found in [4].

\subsection{Pilot-PSI exposures}

Pilot-PSI is a high-density $\left(\mathrm{n}_{\mathrm{e}}=0.1-10 \times 10^{20} \mathrm{~m}^{-3}\right)$, high-flux $\left(>10^{23} \mathrm{~m}^{-2} \mathrm{~s}^{-1}\right)$ linear plasma generator [6] that operates in an axial magnetic field (0.4-1.6T). While intense, the plasma column generated is also quite narrow with a typical ion flux full-width-half-maximum of $\sim 10$ 
$\mathrm{mm}$. Pilot-PSI can be operated in short pulses allowing it to closely re-create many of the conditions experienced by the W Langmuir probe in Alcator C-Mod, namely high density and recycling, high plasma flux, short pulses with transient surface temperatures. The tungsten target exposed was 99.95 at.\% polycrystalline tungsten disk with a diameter of $25 \mathrm{~mm}$ and thickness of $1 \mathrm{~mm}$. The $\mathrm{W}$ target was polished but unannealed before exposure in Pilot-PSI. The exposure consisted of 14 consecutive discharges, each $1.5 \mathrm{~s}$ in duration. Since surface temperature is such a critical parameter for fuzz growth, the plasma conditions were selected to best reproduce the surface temperature magnitude and evolution of the $\mathrm{W}$ probe in Alcator C-Mod. The central flux in the plasma column was $1.5 \pm 0.5 \times 10^{24} \mathrm{He}^{+} / \mathrm{m}^{2} \mathrm{~s}$ at a target bias of $-40 \mathrm{~V}\left(\mathrm{E}_{\mathrm{He}^{+}}=40 \mathrm{eV}\right)$ and a magnetic field of $0.8 \mathrm{~T}$. This resulted in a central maximum surface temperature of $2700 \pm 200 \mathrm{~K}$. The maximum surface temperature as a function of plasma column radius can be seen in Fig. 2a. The surface temperature time evolution of the CMod W probe is similar to that of the Pilot-PSI exposure conditions although surface temperatures in Pilot-PSI exceeded those obtained in C-Mod (see Fig. 2b). A fast infrared camera (FLIR SC7500MB) is used to measure the 2D surface temperature profile at a framerate of $100 \mathrm{~Hz}$.

\subsection{Heavy ion elastic recoil detection}

The 1.7 MV tandem ion accelerator of the Cambridge Laboratory for Accelerator Study of Surfaces (CLASS) is used to accelerate to perform elastic recoil detection (ERD) of He atoms contained in the $\mathrm{W}$ fuzz layers. The beam species selected was oxygen-16 (O) ions. The experimental geometry follows the common forward recoil geometry of ERD with an angle of incidence of 75 degrees from the normal to the surface of the samples and a scattering angle of 30 degrees. For most of the work presented in this paper a $4 \mathrm{MeV}^{16} \mathrm{O}^{3+}$ beam was used with a $3.2 \mu \mathrm{m}$ aluminium stopping foil in front of the detector to filter out the scattered oxygen ions. SIMNRA [7] is a program that simulates ion beam analysis spectra. By re- 
creating the energy spectrum of the forward-scattered He atoms from our ERD measurements in SIMNRA, we determine the He concentration in the $\mathrm{W}$ fuzz layer.

\section{Results and discussion}

\subsection{Alcator C-Mod Exposures}

The Alcator C-Mod exposures were performed on the final day of an Alcator C-Mod run campaign. This helped ensure that the surfaces were not further modified by additional experiments before they were removed and examined. Upon the removal of the ramped tile section from Alcator C-Mod, visual inspection revealed that the $\mathrm{W}$ Langmuir probe at the outer strike point was optically black (figure 3a), which often indicates the growth of nanotendrils on the surface. Inspection of the same W probe tip with a JEOL-6320FV SEM shows fully developed nano-tendrils with almost complete coverage of the surface (figure $3 \mathrm{~b}$ ). A higher magnification image shows the individual $\mathrm{W}$ nano-tendrils are typically $100 \pm 10 \mathrm{~nm}$ in diameter (figure 3c).

There is no evidence of the nano-tendrils melting or damage in the SEM images, demonstrating that despite what is assumed to be a strongly reduced thermal conductivity through the fuzz layer [8], these nano-tendrils can survive and even grow in steady thermal heat fluxes of up to $\sim 40 \mathrm{MW} / \mathrm{m}^{2}$. During the 14-discharge sequence there were three full current $(900 \mathrm{kA})$ disruptions, including on the final shot of the discharge sequence, which had no obvious effect on the fuzz. However, we do not have measurements of the heat flux received by the $\mathrm{W}$ probe during these disruptions. There is also no evidence of the uni-polar arcing that was seen on nano-tendril surfaces exposed in the LHD stellerator [9] and in NAGDIS-II [10].

\subsection{Comparison of Alcator C-Mod results to PISCES and Pilot-PSI}

A systematic study of W fuzz layers grown in PISCES linear plasma device has resulted in an empirical growth formula [1]. Using this $\mathrm{t}^{1 / 2}$-dependent growth rate formula from 
Baldwin et al [1] we can calculate the predicted W fuzz layer depth expected from the Alcator C-Mod exposure. However, since the $\mathrm{W}$ Langmuir probe is being actively biased and the outer strike point in Alcator C-Mod is a region of net erosion, there is a finite amount of sputtering expected on the surface of the W Langmuir probe. The W Langmuir probe was scanning with a bias voltage from $-150 \mathrm{~V}$ to $+50 \mathrm{~V}$ (with respect to the grounded walls) during the exposures. Therefore the potential drop from the plasma to the $\mathrm{W}$ Langmuir probe is $3 \mathrm{~T}_{\mathrm{e}}-\mathrm{V}_{\text {bias. }}$ In determining the energy of incident He ions the inherent energy of the ion $\left(T_{i}=T_{e}\right)$ must be also accounted for, therefore, for a typical divertor $T_{e}$ of $\sim 25$ the $\mathrm{He}^{+}$ion energy will be between $50-250 \mathrm{eV}$ on the $\mathrm{W}$ Langmuir probe. With the ion energy and ion flux (directly from the $\mathrm{W}$ Langmuir probe data) well known as a function of time, the gross sputtering of the $\mathrm{W}$ probe can be calculated using the formula from Eckstein [11]. For sputtering calculations a 90:10 distribution between $\mathrm{He}^{+}$and $\mathrm{He}^{++}$ions and a 1 at.\% population of $\mathrm{B}^{3+}$ in the plasma is assumed.

Using the empirical growth formula from Baldwin et al. [1] and the Eckstein formula for gross sputtering of $\mathrm{W}$ we can calculate the progression of the predicted $\mathrm{W}$ fuzz layer depth and amount of gross sputtering of bulk W as a function of time through the C-Mod discharge sequence (see Fig. 4). We find that after the full 14 discharges, the Baldwin growth formula predicts a fuzz layer depth of $515 \mathrm{~nm}$ and the Eckstein sputtering formula predicts gross sputtering of $35 \mathrm{~nm}$ bulk W. However, in plasma conditions in the C-Mod divertor we expect prompt redeposition of sputtered atoms, meaning the net $\mathrm{W}$ erosion will be much less than the calculated gross sputtering rate and thus we don't expect sputtering to be playing a significantly inhibiting role in the fuzz growth during the Alcator C-Mod exposures.

To compare this prediction to the $\mathrm{W}$ fuzz layer grown in Alcator C-Mod, the $\mathrm{W}$ probe fuzz layer depth was directly measured by focused ion beam cross-section and SEM inspection to be $600 \pm 150 \mathrm{~nm}$. This layer depth is in-line with the calculations from the 
Baldwin formula and proof that a significant layer of W fuzz can grow over a short timescale. It must be mentioned that the Baldwin formula is based on data only at surface temperatures of $1120 \mathrm{~K}$ and $1320 \mathrm{~K}$. Some work has shown this formula to overestimate growth rates for $\mathrm{T}_{\text {surf }}>1400 \mathrm{~K}[3,12]$. It has been suggested that a factor dependent on He ion energy might be missing from the formula [12] and that re-crystallization effects might be competing with the surface changes at higher temperatures [3]. Despite concerns about the formula raised in the literature, the comparison with the Alcator C-Mod results, which were also performed at $\mathrm{T}_{\text {surf }}>1400 \mathrm{~K}$, and the Baldwin formula resulted in good agreement. It is also worth noting that the data for the Baldwin formula [1] as well as from [3] and [12] were taken at steady-state surface temperatures over one continuous plasma exposure while the CMod exposures had transient surface temperatures and were the integration of 14 short discharges.

To better understand the role of transient surface temperatures and multiple short pulses (as opposed to a single continuous discharge), the Alcator C-Mod exposure conditions were re-created as closely as possible in the linear plasma device, Pilot-PSI. After a sequence of 14 x $1.5 \mathrm{~s}$ discharges with slightly higher central surface temperatures but similar temperature evolutions as was calculated for the W probe in Alcator C-Mod (see Fig. 2b), there was obvious surface modification of the Pilot-PSI W target. Since the maximum surface temperature is dependent on radial position for Pilot-PSI exposures (see Fig. 2a), SEM investigation of different radial positions yields a temperature dependence of the surface morphologies and sub-surface structures with focussed ion beam cross-sectioning. In all radial positions the expected ion flux is well in excess of $10^{22} \mathrm{He} / \mathrm{m}^{2} \mathrm{~s}$, which has been shown the be the low-end threshold for optimized fuzz growth [13]. The SEM investigation of different radial positions on the Pilot-PSI W target shows a strong dependence of the characteristic diameter of individual nano-tendrils with tendril diameter increasing strongly with increasing 
surface temperature. Similarly, the diameter of the largest sub-surface voids also increases with a very similar dependence to surface temperature (see Fig. 5). A temperature dependence on the surface morphology has also been observed by other experiments [14]. The very similar surface temperature dependence between the nano-tendril thicknesses and the void diameters seems to indicate a strong relationship or inter-dependence between the nanotendrils and the sub-surface voids.

The Pilot-PSI W target was successfully able to grow a wide range of W fuzz surface morphologies with transient surface temperatures and short pulses similar to the conditions in C-Mod. The closest match to the Alcator C-Mod W fuzz morphology (e.g. nano-tendril thickness of $\sim 100 \mathrm{~nm}$, maximum void diameter $\sim 30 \mathrm{~nm}, \mathrm{~W}$ fuzz layer thickness $\sim 600 \mathrm{~nm}$ ) is found on the Pilot-PSI $\mathrm{W}$ target at a location $\sim 5 \mathrm{~mm}$ radially off-center, which has a maximum surface temperature of $1850 \pm 200 \mathrm{~K}$ (see Fig. 6). This is in agreement, within uncertainties, with the maximum surface temperature of the C-Mod W probe of $2200 \pm 300$ K. This strongly indicates that if the plasma and surface conditions are equivalent (e.g. plasma flux, plasma time/fluence, erosion/depsotion rates, and surface temperature and evolution), then the $\mathrm{W}$ fuzz growth and development will be nearly identical regardless of most other differences between a linear plasma device environment and a tokamak environment.

From these two experiments there are some clear differences when compared with those with longer exposure times and steady-state surface temperatures (e.g. [1-3]). The main difference is in the comparison of the surface morphologies for the maximum surface temperatures in the transient experiments (Alcator C-Mod and Pilot-PSI) and the equivalent steady-state surface temperatures from other experiments. Through experiments with steadystate surface temperatures it has been well documented that $\mathrm{W}$ nano-tendrils begin to form at $\sim 1000 \mathrm{~K}$, and as surface temperature increases, the nano-tendrils' diameter increases until at $\sim 2000 \mathrm{~K}$ the individual nano-tendrils become indistinct and the surface is populated with 
large pores [10]. For the transient surface temperature exposures on Pilot-PSI, a similar transition from fine to coarse nano-tendrils is seen as the maximum surface temperature rises, finally transitioning to large surface pores at the highest surface temperatures, but the thresholds are different. For example, even when a maximum surface temperature of $\sim 1200 \mathrm{~K}$ is achieved in Pilot-PSI, there is no distinct nano-tendril formation, and at temperatures of $\sim 2200 \mathrm{~K}$, the morphology is coarse but still distinctly visible. Since the surface temperatures are transient, the $\mathrm{W}$ targets are only in the range of these maximum temperatures for a small fraction of the total exposure time. Thus, it is likely, with transient surface temperatures, that the surface morphology is determined by a gradual evolution throughout each temperature cycle rather than simply corresponding to the maximum obtained surface temperature. This indicates that changes in the surface morphology characteristics are not occurring on short time scales $(<0.1 \mathrm{~s})$.

\subsection{He concentrations in $\mathrm{W}$ fuzz layers}

Given the correlation of the nano-tendril thickness with void diameter, the formation of these sub-surface voids is likely playing an important role in the growth of these nanotendrils. Thus it is important to further investigate the possible mechanisms of the He void formation.

One possible mechanism for the formation of these voids is the accumulation of $\mathrm{He}$ inside a seed site (e.g. a lattice vacancy) until such high pressures are obtained that loop punching can occur [15]. This is a pressure driven mechanism and typical pressures required for loop-punching are in the $5 \mathrm{GPa}$ range for most metals [16]. Even after the He implantation has ceased, the equilibrium He pressure is expected to be very high. If we assume temperature of $1350 \mathrm{~K}$ as an example where we know nano-tendrils grow well, then we can estimate the equilibrium He pressure in the $10 \mathrm{~nm}$ diameter bubbles (see Fig. 5) at $\sim 1 \mathrm{GPa}$ using the same method as was used in [12]. From a simple ideal gas law calculation, we can estimate the 
density of the He in that void to be $\sim 5.4 \times 10^{28} \mathrm{He} / \mathrm{m}^{3}$, or $\sim 85 \%$ of the number density of the surrounding tungsten. Given that the porosity (e.g. bubble/void density in the solid tendrils or bulk) of the tungsten filled with these He voids has been measured between $20-50 \%$ porous $[17,18]$, we would then expect the He concentrations in these layers to be in the range of 1743 at. $\%$.

Using heavy-ion elastic recoil detection we can measure the He concentrations in these nano-tendril layers directly. This has been performed over a wide variety of $\mathrm{W}$ targets exposed in Pilot-PSI and PISCES. The Pilot-PSI W target from the C-Mod re-creation exposures was measured as well as other targets exposed in Pilot-PSI to similar plasmas but with one steady-state discharge ranging from 10-60 s. The PISCES targets were also a steadystate discharge but for much longer exposure times (300-3000 s). Regardless of surface temperature, ion flux density or fluence, or the presence of nano-tendrils, the $\mathrm{He}$ concentration for all targets is measured in the range of 1-4 at.\% (see Fig. 7). This is well below the expected He concentration if the voids were filled to equilibrium pressure with $\mathrm{He}$ gas. It is also possible that the void fraction in the tendrils and bulk was significantly lower than in the $\mathrm{W}$ in [17] and [18]. However, we can think of no reason this might be the case given the consistent morphology of fuzz grown in Pilot-PSI when compared to other devices. Also the SEM images of the cross-section (Fig. 6d for example) do show significant void fraction even when not including smaller voids $(<5 \mathrm{~nm})$ that are only visible with TEM. These high void densities can also lead to interconnected void structures as the voids expand. This can create pores out to the surface and thus release the contained $\mathrm{He}$, which is another possible explanation of the low He concentrations measured in this work. Unfortunately, before we could measure the He concentration of the Alcator C-Mod W probe, the surface became compromised by a contaminant. In an attempt to remove the contaminant via plasma exposure in a helicon plasma source, the $\mathrm{W}$ probe surface was damaged by arcing within the 
helicon plasma. The He content of the surface was measured with the contaminant and after the arcing damage as $0.20 \pm 0.05$ at.\% for both measurements. Unfortunately, there are no measurements of the true He concentration of the nano-tendrils grown in Alcator C-Mod.

Still, it is important to realize that while the He concentration found in the fuzz layer is well in excess of the natural solubility of $\mathrm{He}$ in $\mathrm{W}$, it is still well below what would be expected if the voids were filled with $\sim 1 \mathrm{GPa}$ of $\mathrm{He}$ gas and the void fraction is similar to what has been measured in other $\mathrm{W}$ fuzz targets. We also see that while the void volumes are increasing with surface temperature (see Fig. 5), the total He concentration in the $\mathrm{W}$ is not. Since these measurements are only post-mortem it does not conclusively rule out the pressure driven void formation. It would seem that some of the He would need to be released from the $\mathrm{W}$ targets after the plasma exposure for the pressure-driven void formation to be consistent with these measurements. He does outgas from $\mathrm{W}$ at temperatures $>1000 \mathrm{~K}$ [17] so there could be some outgassing as the targets cool back down to $<1000 \mathrm{~K}$, but for Pilot-PSI targets, which are actively water cooled, the temperature is reduced to $<1000 \mathrm{~K}$ very quickly. A measurement of the He concentration of these layers as they are dynamically forming would help resolve many of these uncertainties.

\section{Summary}

The defining result of this work is that $W$ fuzz can be grown on surfaces in a tokamak divertor. These structures not only survive under the intense heat flux of up to $\sim 40 \mathrm{MW} / \mathrm{m}^{2}$ and transient conditions of the Alcator C-Mod lower divertor, but grow in these conditions. The layer thickness of this W fuzz layer is compared to predictions made with the empirical formula from Baldwin et al [1] and found to be in good agreement despite the high surface temperatures of the Alcator C-Mod W probe. To investigate W fuzz growth at higher temperatures with short plasma pulses and dynamic surface temperatures the Alcator C-Mod experiment was re-created in Pilot-PSI. W fuzz, nearly identical in terms of surface 
morphology and layer thickness to that found on the C-Mod W probe, was found on the $\mathrm{W}$ target exposed in Pilot-PSI, at surface temperatures of $1850 \pm 200 \mathrm{~K}$, which is in agreement within uncertainties with the $2200 \pm 300 \mathrm{~K}$ the C-Mod W probe achieved. This agreement indicates that $\mathrm{W}$ nano-tendril formation and morphology is nearly identical in a linear plasma device environment and a tokamak environment. This has significant impact in justifying the use of data, modelling and simulations based on linear plasma device experiments for making predictions towards W nano-tendril growth and morphology in future fusion devices. Finally, He concentrations in the $\mathrm{W}$ fuzz layer were measured to be $1-4$ at.\% in a wide range of exposure conditions in Pilot-PSI and PISCES. This He concentration is well below what one would expect if all observable sub-surface voids were filled to equilibrium He pressure and void fraction is similar to that measured on other W fuzz targets. This creates some uncertainty as to the validity of the pressure-driven loop-punching model for the formation and growth of these sub-surface voids.

\section{Acknowledgements}

The authors would like to thank the Alcator C-Mod team for their time and assistance. This work is supported by US DOE award DE-SC00-02060. This work made use of the MRSEC Shared Experimental Facilities at MIT, supported by the National Science Foundation under award number DMR-08-19762. Alcator C-Mod is supported by US DOE contract DE-FC02-99ER54512.

\section{References}

[1] M.J. Baldwin, R.P. Doerner, Nucl. Fusion 48 (2008) 035001.

[2] S. Takamura, N. Ohno, D. Nishijima, S. Kajita, Plasma Fusion Res. 1 (2006).

[3] G. de Temmerman et al., J. Vac. Sci. Technol. A 30 (2012) 041306.

[4] G.M. Wright, D. Brunner, M.J. Baldwin, R.P. Doerner, B. Labombard, B. Lipschultz, J.L. Terry, D.G. Whyte, Nucl. Fusion 52 (2012) 042003.

[5] E. Marmar and the Alcator C-Mod Team, Fusion Sci. Technol. 51 (2007) 261. 
[6] G. J. van Rooij, V. P. Veremiyenko, W. J. Goedheer, et al., Appl. Phys. Letters 90 (2007) 121501.

[7] M. Mayer, Technical Report IPP 9/113, Max-Planck-Institut für Plasmaphysik, Garching, Germany (1997).

[8] S. Kajita, S. Takamura, N. Ohno, D. Nishijima, H. Iwakiri, N. Yoshida, Nucl. Fusion 47 (2007) 1358 .

[9] M. Tokitani, S. Kajita, S. Masuzaki, Y. Hirahata, N. Ohno, T. Tanabe, Nucl. Fusion 51 (2011) 102001.

[10] S. Kajita, S. Takamura, N. Ohno, Nucl. Fusion 49 (2009) 032002.

[11] W. Eckstein, J. Nucl. Mater. 248 (1997) 1.

[12] S. Kajita, N. Yoshida, R. Yoshihara, N. Ohno, M. Yamagiwa, J. Nucl. Mater. 418 (2011) 152.

[13] M.J. Baldwin, R.P. Doerner, D. Nishijima, K. Tokunaga, Y. Ueda, J. Nucl. Mater. 390391 (2009) 886.

[14] Sakaguchi W., Kajita S., Ohno N., Takagi M. and Kurishita H. 2008 Proc. 18th Int. Toki Conf. (Toki, Japan, 9-12 December 2008) P1.29.

[15] J.H. Evans, A. van Veen, L.M. Caspers, Scripta Metallurgica 15 (1981) 323.

[16] J.B. Condon, T. Schober, J. Nucl. Mater. 207 (1993) 1.

[17] S. Kajita et al. J. Nucl. Mater. 421 (2012) 22.

[18] M. Miyamoto, D. Nishijima, M. Baldwin, R. Doerner, Y. Ueda, K. Yasunaga, N.

Yoshida, K. Ono, J. Nucl. Mater. 415 (2011) S657. 


\section{Figure captions:}

Figure 1: a) Surface heat flux and b) surface temperature for the $\mathrm{W}$ probe during a plasma discharge. The solid lines represent the shot with the median heat flux/temperature for the 14shot sequence. The dashed lines represent the shots with the maximum and minimum heat flux/temperature for the 14-shot sequence.

Figure 2: a) the maximum surface temperature of the $\mathrm{W}$ target exposed in Pilot-PSI as a function of radial position with respect to the center of the plasma column, and b) a comparison of the time evolution of the surface temperatures for the center of the Pilot-PSI W target and the Alcator C-Mod W probe.

Figure 3: a) The ramped Mo tiles and W probe upon removal from Alcator C-Mod, b) SEM image of the $\mathrm{W}$ probe surface showing nano-tendrils on the surface, and c) high magnification SEM image.

Figure 4: The time evolution of the calculated depth of the $\mathrm{W}$ fuzz layer according to the empirical formula by Baldwin et al [1] for the Alcator C-Mod 14-discharge sequence and the calculated gross sputtering of bulk W over the same discharge sequence. The measured $\mathrm{W}$ fuzz layer thickness from focussed ion beam cross-sectioning is also plotted.

Figure 5: The characteristic W nano-tendril thickness and maximum sub-surface void diameter as a function of maximum surface temperature for the Pilot-PSI W target.

Figure 6: a) Surface nano-tendrils with characteristic thickness of $\sim 100 \mathrm{~nm}$ from the Alcator C-Mod W probe, b) surface nano-tendrils with characteristic thickness of $\sim 100 \mathrm{~nm}$ from the Pilot-PSI W target, c) cross-section of the W fuzz from the Alcator C-Mod W probe shown in a), and d) cross-section of the $\mathrm{W}$ fuzz from the Pilot-PSI $\mathrm{W}$ target shown in $\mathrm{b}$ ).

Figure 7: Helium concentration in the surface morphology layer for various targets exposed in Pilot-PSI and PISCES-A as a function of surface temperature. 
Figure 1:

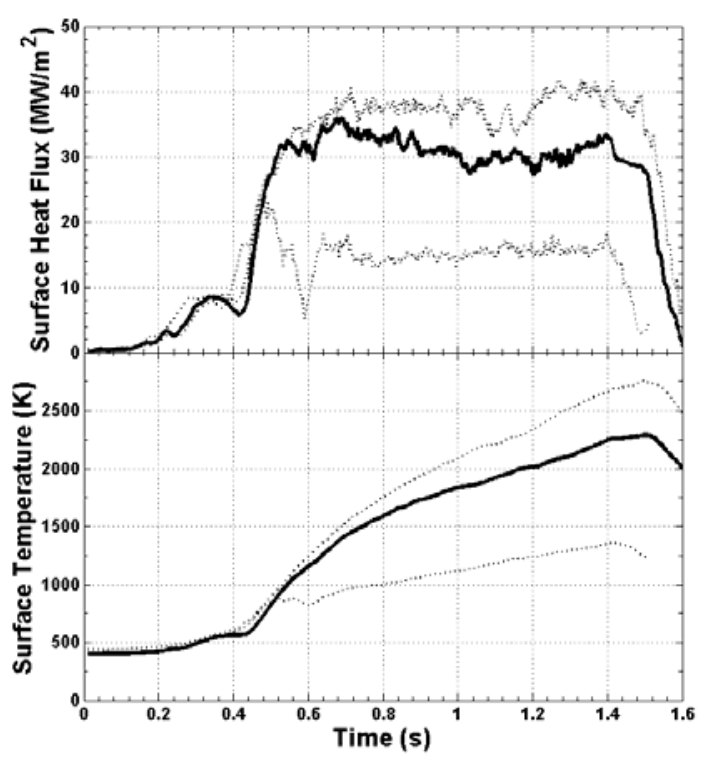


Figure 2:
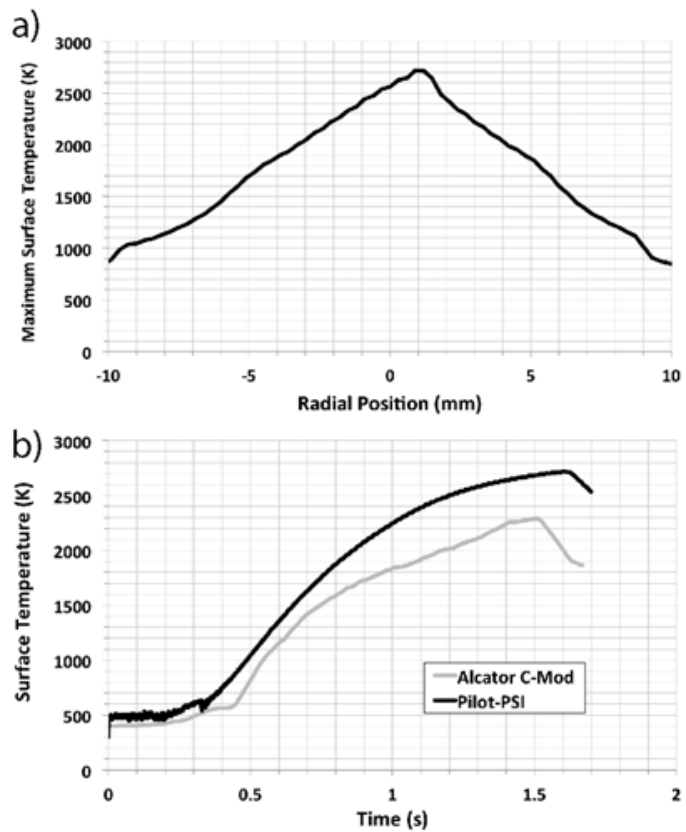
Figure 3:
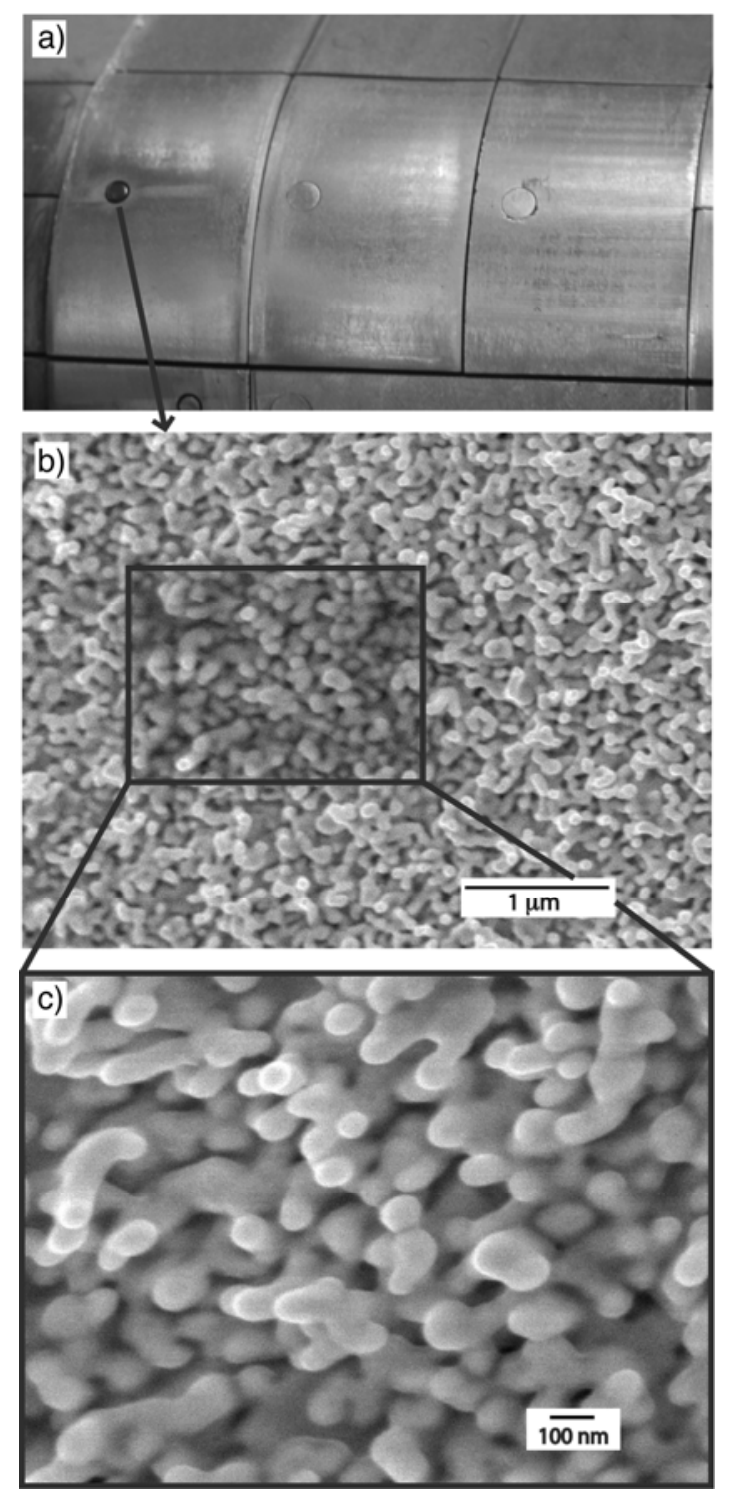
Figure 4:

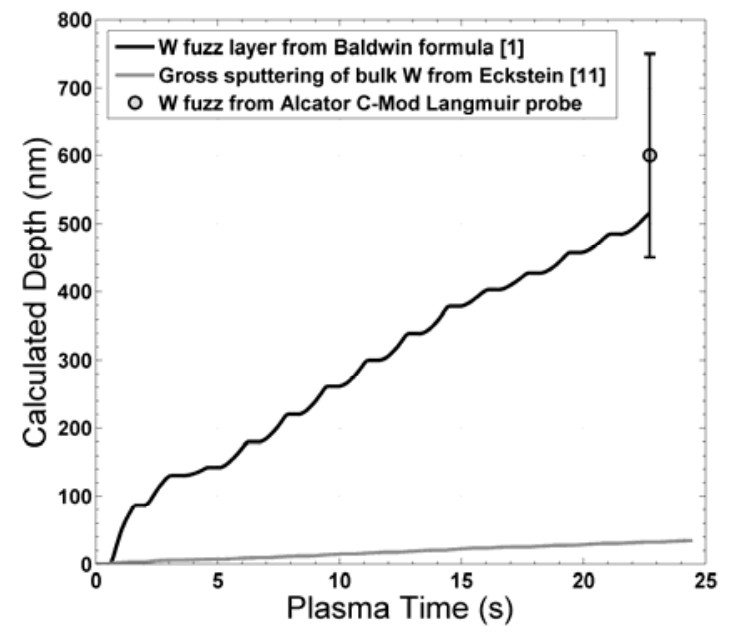


Figure 5:

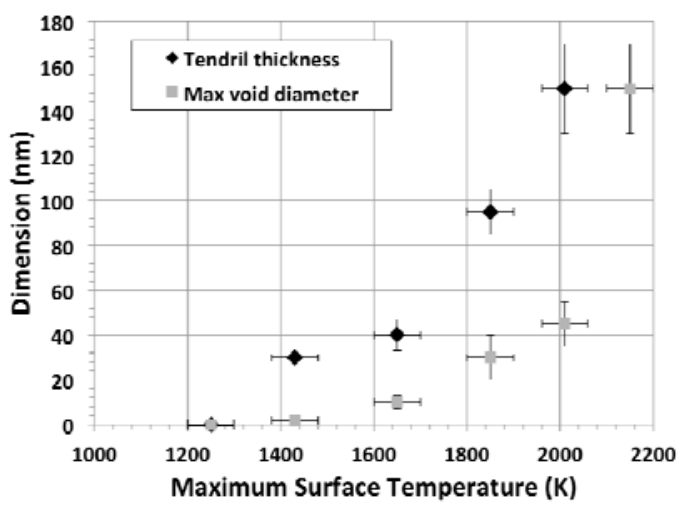


Figure 6:
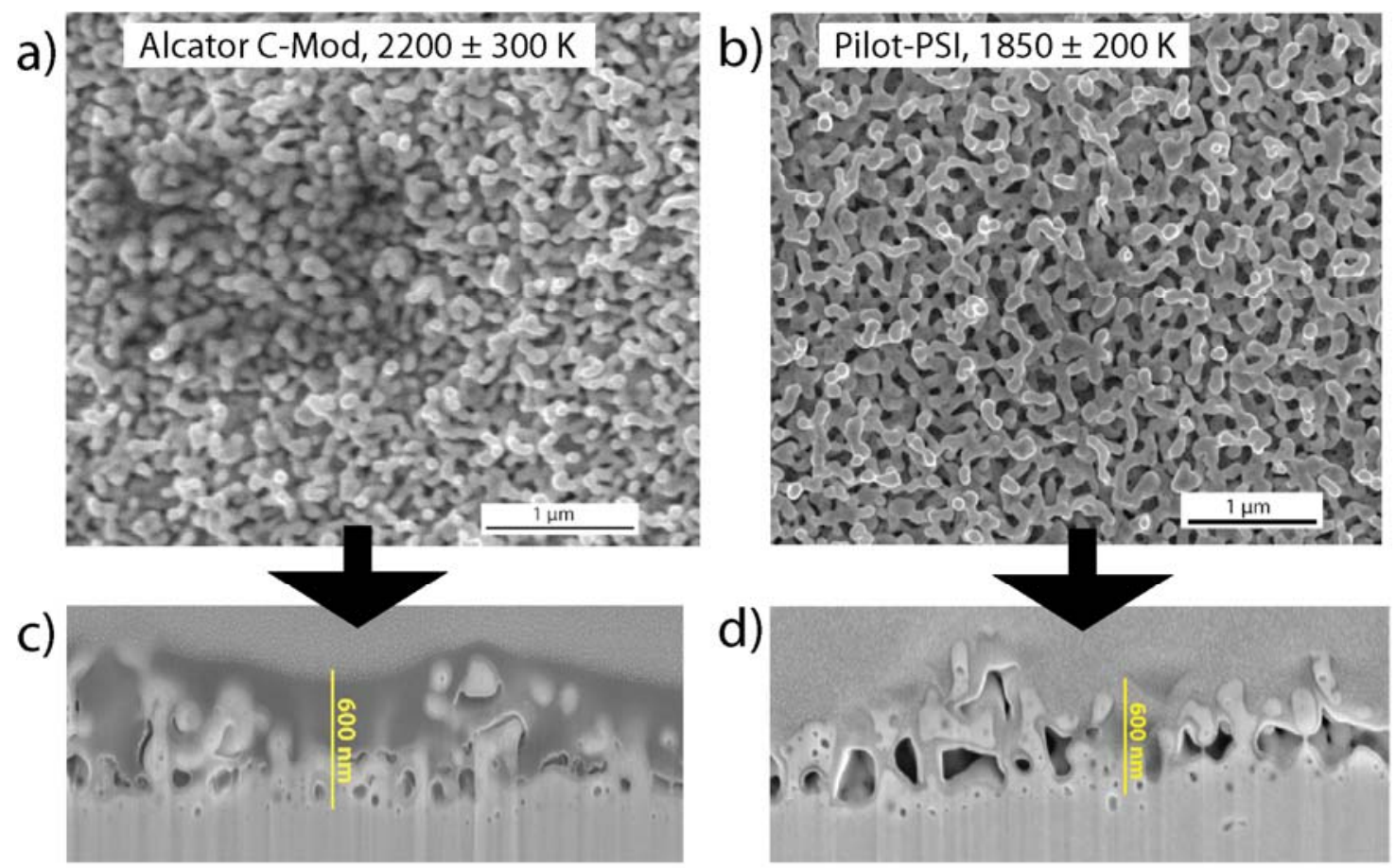
Figure 7:

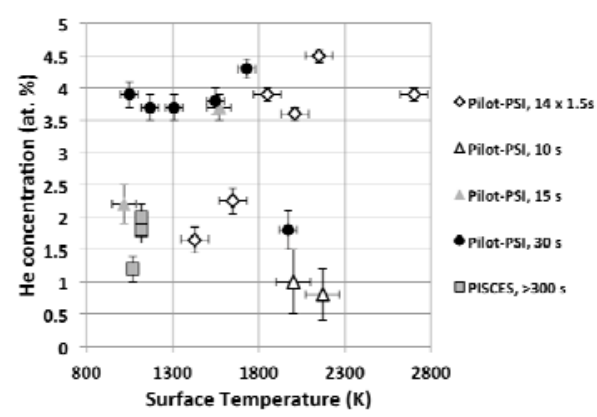

